\title{
BIOMECÁNICA EN EL CONTROL MOLAR
}

\author{
Juan Esteban Rendón Giraldo*, Odon. ${ }_{1}$, Gabriel Castaño, Odon. ${ }_{2}$ \\ ${ }_{1}$ Universidad de Antioquia, Universidad Cooperativa de Colombia, sede Medellin, Colombia \\ ${ }_{2}$ Universidad CEs, Universidad de Antioquia, Universidad Cooperativa de Colombia, sede Medellín, Colombia
}

Recibido: 10 de diciembre del 2012 Aprobado: 8 de marzo del 2013

* Autor de contacto: Juan Esteban Rendón, Facultad de Ortodoncia, Universidad Cooperativa de Colombia, 2706466, Carrera 47\# 37 sur 18, Medellín, Colombia, correo electrónico: juanesregi@hotmail.com

Cómo citar este artículo: Rendón Giraldo JE, Castaño G. Biomecánica en el control molar. Rev. Nac. Odontol. 2013 diciembre; 9 (edición especial): 109 -117.

Resumen. Una de las dificultades que surge durante la mecánica de tratamiento es el inadecuado control molar, el cual, tratado de una manera eficiente, permite un control preciso de fuerzas y de momentos, lo que aumenta la eficacia de la biomecánica. El conocimiento de los sistemas parciales 4 × 2, 6 × 2 y la barra traspalatina con sus diferentes formas de activación, dan una serie de ventajas que permiten la optimización de los resultados ortodóncicos. El objetivo de este artículo es aclarar conceptos, detallar posibles estrategias de tratamiento y analizar los efectos secundarios que se presentan con estos sistemas.

Palabras clave: biomecánica, barra traspalatina, momentos diferenciales, sistemas parciales.

\section{Biomechanics in Molar CONTROL}

Abstract. Inadequate molar control is one of the difficulties that arise during mechanical treatment. When molar control is handled efficiently, it facilitates precise control of forces and moments, which increases the effectiveness of biomechanics. Knowledge of $4 \times 2$, $6 \times 2$ partial systems and the transpalatal bar with its different forms of activation offer a number of advantages that make it possible to optimize orthodontic results. The aim of this article is to clarify concepts, specify possible treatment strategies and analyze the side effects that occur with these systems.

Keywords: biomechanics, transpalatal bar, differential moments, partial systems. 


\section{Introducción}

Para el ortodoncista no es fácil reconocer las múltiples oportunidades que ofrece el control molar a nivel clínico, llevándolo por tanto a emplear aparatos auxiliares que pueden disminuir la eficacia de la higiene oral, favorecer la inflamación gingival o la aparición de problemas mucogingivales [1], y resultar en sobrecostos del tratamiento. Cuando el ortodoncista entiende la utilidad del control molar, descubre que muchos de estos aparatos no son tan necesarios y al mismo tiempo simplifica la mecánica sin dejar de obtener resultados óptimos. Esto no significa que se deban desechar los aparatos auxiliares, por el contrario, el conocimiento de la biomecánica le permitirá identificar en qué casos son útiles. Es importante recalcar que los principios mecánicos no han cambiado, situación que otorga al clínico la capacidad de elegir el tipo de sistema más eficiente que le otorgue mayores posibilidades de éxito y control del movimiento dental.

Por lo descrito anteriormente, la intención de este artículo es aclarar conceptos y explicar estrategias de tratamiento que incluyen el análisis de los efectos secundarios con sistemas parciales y con la barra traspalatina para orientar al ortodoncista en el manejo biomecánico que ofrece el control molar facilitando su práctica clínica.

Existen casos con malposiciones de un diente o un grupo de dientes que pueden resultar en maloclusiones simétricas o asimétricas [2], las cuales - cuando son relativamente pequeñas- se pueden corregir con sistemas parciales o con un simple aditamento - como la barra traspalatina- que, al estar bajo el control del ortodoncista no dependen de la colaboración del paciente, como cuando se utilizan aparatos removibles $[3,4]$.

Para la activación de este tipo de sistemas deben estar claros conceptos como la cupla y el momento. Una cupla consiste en un par de fuerzas iguales y opuestas que producen una tendencia a la rotación alrededor del centro de resistencia de un diente en cualquier plano del espacio. Un momento se produce cuando una fuerza actúa lejos del centro de resistencia de un diente o un grupo de dientes y se determina al multiplicar la magnitud de la fuerza por la distancia perpendicular que hay desde el punto de aplicación hasta el centro de resistencia de un cuerpo [5]. Es importante reconocer que diferentes magnitudes de fuerza pueden producir el mismo momento dependiendo de la longitud al centro de resistencia, lo cual es significativo en ortodoncia porque ofrece la oportunidad de producir momentos deseables sin ejercer grandes magnitudes de fuerza [6], tratando de mantener los niveles de tensión bajos y uniformes en el ligamento periodontal [7-11]. Teniendo despejadas estas nociones, a continuación se describen las bases biomecánicas de los sistemas parciales y de la barra traspalatina para facilitar su uso clínico.

\section{Sistemas parciales $(4 \times 2$ y 6 × 2$)$}

Cuando se trabaja con un sistema 4 × 2 y 6 × 2 se tienen dos grupos de dientes, los primeros molares permanentes, y el grupo de dientes anteriores, en el que los dientes anteriores son considerados como un diente multirradicular con un mismo centro de resistencia.

Debido a la distancia entre ambos grupos de dientes se pueden ubicar dobleces de manera precisa, lo cual permite ejercer fuerzas y momentos más duraderos, o sea una respuesta directa, gracias a que no tiene las limitaciones de movimiento que existe cuando la distancia interbracket es corta como en un sistema completo, en el que los momentos generados son casi iguales y opuestos con fuerzas de equilibrio que se cancelan entre sí anulando el movimiento que se desea [12-14]. La ventaja de estos sistemas es que, al contar con una mayor longitud de alambre, permite realizar cualquier activación, ya sea en sentido vertical o transversal, resultando en una baja tasa carga deflexión con un amplio rango de actividad y entrega de fuerzas constantes [14-16].

Cabe anotar que es difícil calcular la magnitud de las fuerzas y los momentos que se desarrollan con este tipo de activaciones, pero aunque son sistemas estáticamente indeterminados, el análisis clínico permite determinar los momentos generados y la dirección de las fuerzas de equilibrio presentes en el sistema, por tanto se puede predecir hacia donde se van a mover los dientes $[5,17,18]$, y también reconocer que tanto las fuerzas horizontales como las verticales pueden producir movimientos en el plano frontal [19], como por ejemplo: una fuerza lingual a través de un tubo resulta en un momento lingual de la corona, mientras que una fuerza bucal aplicada en el mismo tubo produce un momento bucal de esta. Por otro lado, fuerzas intrusivas crean el potencial para inclinar la corona hacia vestibular, mientras que fuerzas extrusivas la inclinan hacia lingual, aunque por lo general esta última no ocurre gracias a las fuerzas oclusales, que a pesar de ser transitorias son de mayor magnitud $[2,4,6]$. Lo que sí debe estar claro es que fuerzas horizontales producen momentos más grandes que fuerzas verticales cuando actúan a través del tubo de un molar, ya que la distancia perpendicular entre la fuerza 
horizontal y el centro de resistencia del molar es mayor que la distancia perpendicular entre la fuerza vertical y el mismo centro de resistencia [6].

Para la activación de este tipo de sistemas simplemente se realizan dobleces que simulan el ángulo de entrada que se produce entre el alambre y el bracket cuando existen malposiciones en cualquier plano del espacio, los cuales se conocen como: off center bends (dobleces asimétricos en $\mathrm{V}$ ), step bends (en escalón) y center bends (dobleces simétricos en V) $[6,17,20,21]$.

\section{Off center bend}

Todo doblez en la mecánica ortodóncica puede ser considerado como un off center bend, del cual resultan momentos que pueden ser opuestos - en el mismo sentido, o incluso no existir dependiendo de la ubicación del doblez-, con fuerzas de equilibrio que pueden ser intrusivas o extrusivas en la dimensión vertical, o pueden dirigirse en sentido vestibular o lingual en el plano transversal. Para entender en qué dirección actúa la fuerza, sólo basta con introducir un extremo del alambre en el tubo/bracket, mientras que el otro extremo apunta en el sentido de la fuerza. El bracket localizado cerca del doblez experimentará un momento mayor indicando las fuerzas de equilibrio, y el bracket más retirado tendrá un momento menor que puede ser en sentido horario, antihorario, o nulo, lo cual simplemente depende de la ubicación del doblez [15], o sea, si está centrado, a un tercio, o a menos de un tercio de la longitud.

De un off center bend resulta un tramo largo y uno corto. Cuando el doblez se sitúa a un tercio de la distancia del alambre entre dos dientes no se detecta ningún momento en el bracket más alejado, sólo una fuerza porque el alambre entra pasivo en este. En la medida en que el doblez se aproxime a menos de un tercio a uno de los brackets, la magnitud de los momentos y las fuerzas de equilibrio van variar, mientras que la dirección de los momentos permanece igual (figura 1) [14].

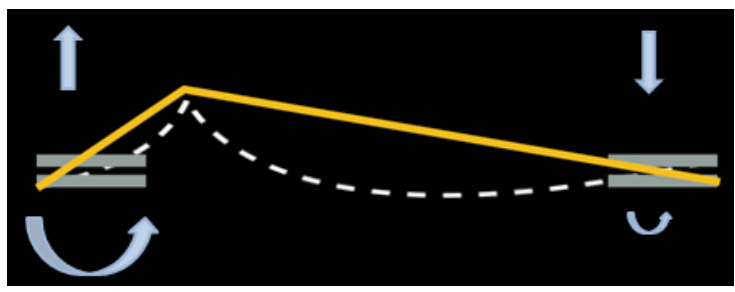

Figura 1. Off center bend ubicado a menos de un tercio Fuente: elaboración propia

\section{Center bend}

El center bend es un doblez que se ubica equidistante entre ambos brackets ofreciendo momentos iguales y opuestos en los que las fuerzas de equilibrio se anulan entre sí (figura 2). En este caso la posición del doblez en $\mathrm{V}$ es una variable que influye de forma trascendental en su efecto, dado que si se altera cambia la geometría a un off center bend alterando la fuerza del sistema $[14,18]$. Un center bend equivale a dos off center bends en los que las secciones cortas se dirigen en la misma dirección, lo cual otorga el mismo sistema de fuerzas, sólo que con la aplicación de dos dobleces en vez de uno existe mayor control del sistema en dos pasos [22]. La ventaja de este tipo de doblez es que ayuda a paralelizar las raíces durante el cierre de espacios sin efectos verticales [23].

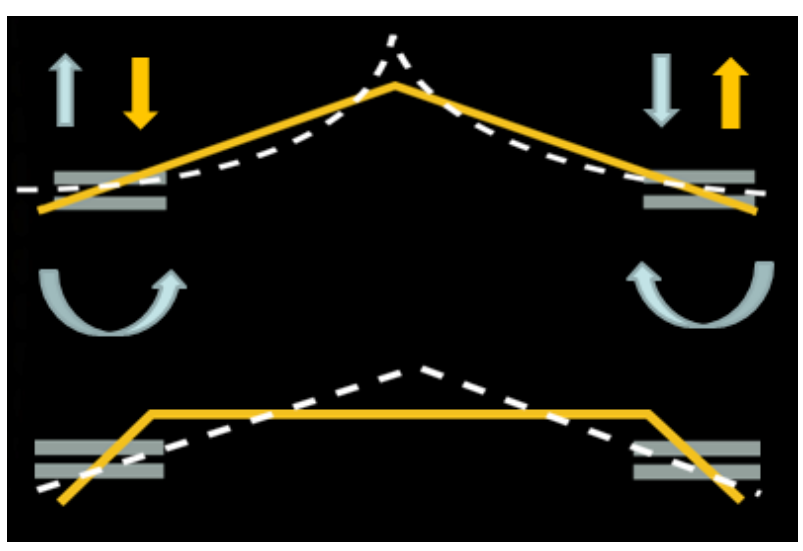

Figura 2. Center bend

Fuente: elaboración propia

\section{Step bend}

El step bend no es más que una combinación de dos off center bend en sentido contrario, en la que los tramos cortos del alambre resultan en direcciones opuestas y paralelas generando dos momentos en la misma dirección y fuerzas de equilibrio que son aditivas [6, 15] (figura 3). Como produce dos fuerzas en la misma dirección, deben aplicarse sólo cuando se desean grandes magnitudes de fuerza [24], por ejemplo para realizar movimientos en el plano sagital en pacientes con el ángulo del plano mandibular disminuido y gran fuerza muscular, o en el plano oclusal cuando los molares deben ser desplazados en sentido transversal para la corrección de mordidas cruzadas. 


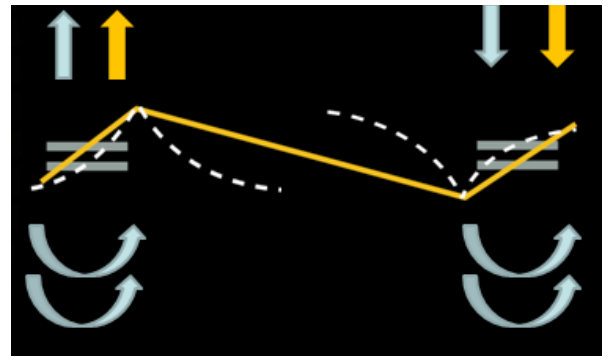

Figura 3. Off center bend

Fuente: elaboración propia

Teniendo en cuenta que el off center bend es clave para los distintos tipos de activaciones, se debe reconocer que las fuerzas y los momentos que genera este doblez son iguales tanto en el plano sagital como en el plano transversal, pero para facilitar su comprensión y aplicación se describen con diferentes términos según el plano del espacio en el que se realice la activación, así: Tip back bend - tip forward bend; Toe in - Toe out; In bend - out bend y In bend - out bend.

\section{Tip back bend - tip forward bend}

Son dobleces de segundo orden en el plano sagital que se aplican al molar, donde el tip back produce un movimiento distal de la corona y mesial de la raíz, mientras que el tip forward genera un movimiento mesial de la corona y distal de la raíz. Al aplicar un tip back, el extremo corto del alambre se dirige en dirección apical, con fuerzas de equilibrio extrusivas en el molar e intrusivas en los dientes anteriores, situación que favorece la corrección de una mordida profunda. También puede ser útil durante el cierre de espacios, ya que produce un momento que resulta en una inclinación radicular mesial, y por tanto el desplazamiento mesial del molar requiere de un movimiento en cuerpo, lo cual resulta en un incremento del anclaje (figura 4). Sucede lo contrario si se realiza un tip forward bend, aunque este doblez es de poca utilidad clínica.

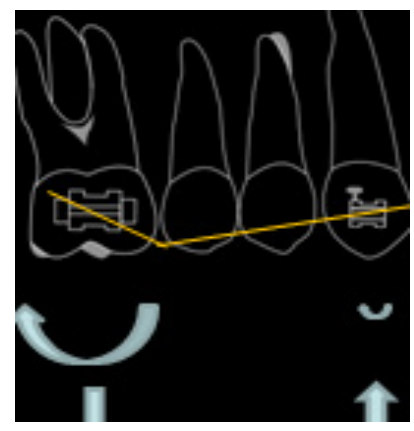

Figura 4. Tip back

Fuente: elaboración propia
Toe in - Toe out

Son dobleces de primer orden actuando sobre el molar en el plano transversal, donde el primero produce una rotación mesial vestibular y el segundo una rotación mesial palatina (figura 5). Estos dobleces, al localizarse justo mesial al tubo del molar, generan un momento rotacional, el cual apunta en dirección opuesta a la fuerza producida hacia bucal en el toe in, y hacia lingual en el toe out. Estos permiten la corrección de mordidas cruzadas leves y la rotación de molares superiores [25] que pueden encontrarse en clase II y además ocupar espacio excesivo que puede ser necesitado en otras partes del arco [26-28]. Un claro ejemplo clínico es cuando se aplica un toe in durante un cierre de espacios para producir un contra momento en el plano transversal, lo cual puede evitar la rotación mesio lingual de los molares [15].
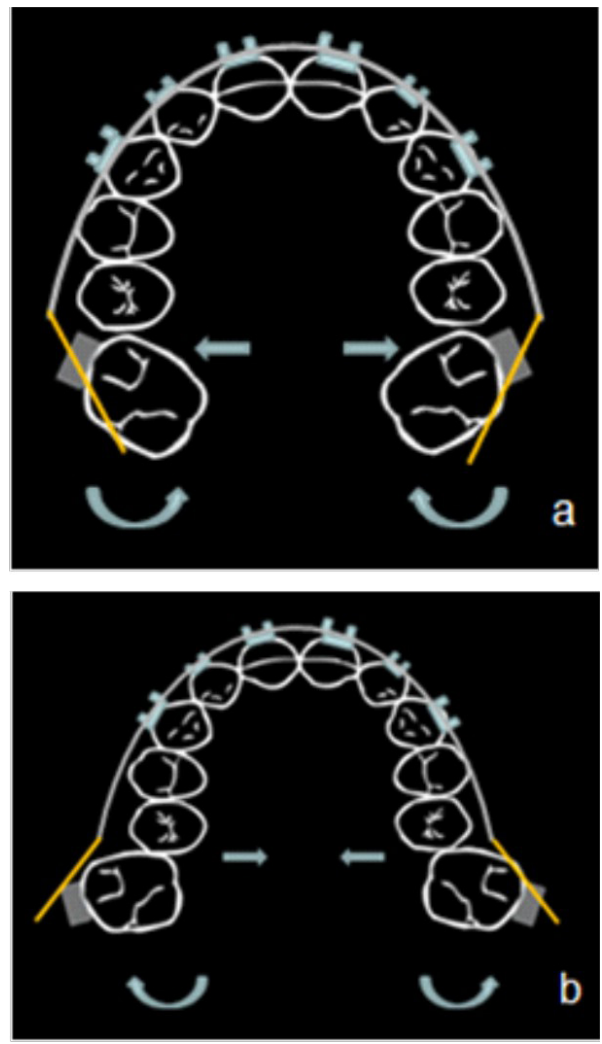

Figura 5. a. Toe in. b. Toe out

Fuente: elaboración propia

In bend - out bend

Este tipo de dobleces se activan en el plano transversal y representan la porción larga del off center bend, la cual apunta en la dirección de la fuerza producida a diferencia de los descritos anteriormente (figura 6). Estos deben estar ubicados en una situación interproximal entre 
caninos y primeros bicúspides si es un sistema $6 \times 2$, o distal a la posible posición de caninos en un sistema 4 x 2. La fuerza que generan estas activaciones actúan a través de los tubos de los molares generando momentos en el tercer orden, lo que puede producir un desplazamiento del molar en dirección bucal o lingual.
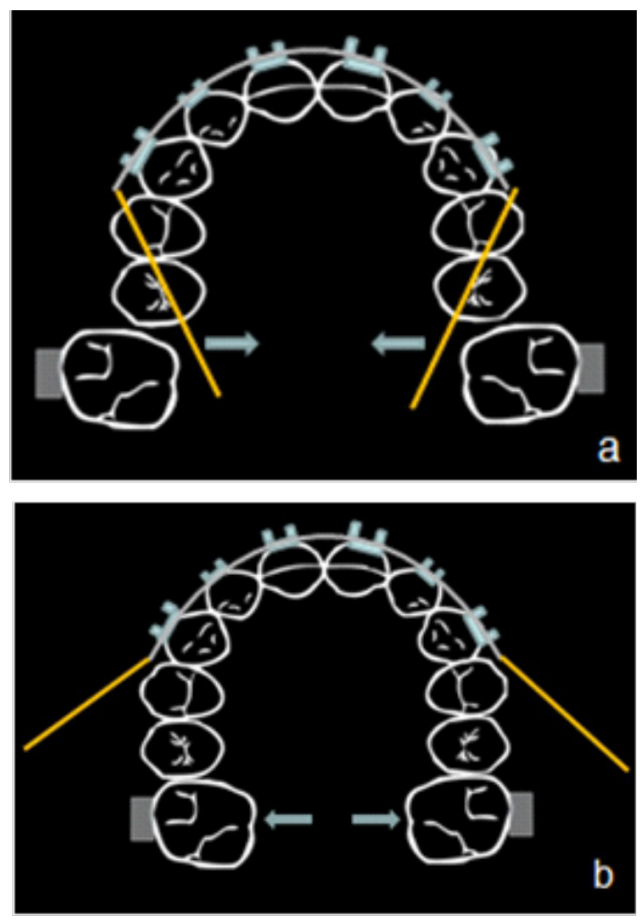

Figura 6. a. In bend. b. Out bend Fuente: elaboración propia

Las cuatro activaciones que se realizan en sentido transversal pueden combinarse para formar ocho tipos diferentes de sistemas de fuerzas que le permiten al clínico mantener la posición del molar o corregirla cuando se pierde. Sin embargo, es importante recordar que toda activación representa una reacción, por ejemplo si se requieren dobleces rotacionales como el toe-in o toe-out, es normal que se presentan fuerzas en sentido bucal o lingual como efecto secundario, que si se quieren controlar sólo basta con aplicar un in-bend o out-bend para proveer fuerzas en dirección opuesta y contrarrestar su efecto.

O sea, existe la posibilidad de crear center bends y step bends a partir de off center bends, así:

- Un off center bend resulta de realizar un toe in, un toe out, un in bend o un out bend.

- Un center bend, es el resultado de aplicar un toe in combinado con un in bend, o, un toe out con un out bend.
- Un step bend puede resultar de combinar un toe in con un out bend, o de un toe out con in in bend, en este caso la orientación de los tramos cortos es opuesta, pero los momentos tendrán la misma dirección y las fuerzas de equilibrio son de mayor magnitud, a diferencia de un off center bend [17]. Estos dobleces son de gran utilidad en la corrección de mordidas cruzadas posteriores, ya que se puede hacer una expansión del arco con la simple aplicación de off center bends (out bend) y step bends (toe in más out bend), sean unilaterales o bilaterales [29, 30 , de lo cual resultan fuerzas y momentos rotacionales en los molares con dirección vestibular.

\section{Barra traspalatina}

Otra opción para ejercer el control molar es la barra traspalatina (вТР) - aditamento desarrollado técnica y clínicamente por el doctod Goshgarian (1972) - la cual permite aplicaciones muy versátiles y puede ser activada de forma simétrica o asimétrica para realizar múltiples funciones, incluyendo estabilización después de una expansión palatina, para incrementar el anclaje [31-34], para la rotación de molares y ganar longitud de arco [35-37], para control del torque e inclinación mesio distal como alternativa para la corrección de la relación molar con una ligera discrepancia clase II [38], para control vertical de los molares impidiendo su extrusión [39-42], para el tratamiento de mordidas cruzadas unilaterales $[43,44]$ y para mantener la forma del arco durante el tratamiento [32].

Entender la biomecánica de la BTP puede ser complejo dado que funciona como un sistema de dos brackets que se ubican en lingual de los molares y que, asociados a un sistema completo, producen un sistema de fuerza estáticamente indeterminado $[18,45$, 46]. La BTP puede ser activada en sus extremos para mover o rotar los molares maxilares en los tres planos del espacio generando momentos de cupla que son de mayor magnitud y se dan en el lugar de la activación, momentos de fuerza con magnitud reducida y fuerzas de equilibrio [15, 47]. A continuación se describen las activaciones y los efectos secundarios que se pueden expresar en el primer orden (plano oclusal), segundo orden (plano sagital) y tercer orden (plano frontal).

\section{Activaciones en el primer orden}

Las activaciones en el primer orden pueden ser simétricas o asimétricas. Cuando son simétricas se producen 
cuplas iguales y opuestas que se traducen en una rotación de los molares alrededor del centro de resistencia (figura 7), aunque clínicamente esto es imposible dado que durante la inserción de los extremos se puede presentar cierta activación cambiando el sistema de fuerzas $[32,48]$. La dirección de la rotación depende de la activación que se le dé a los extremos de la barra, en este caso no hay movimientos anteroposteriores porque las fuerzas de equilibrio, al ser iguales y opuestas, se anulan entre sí $[15,35,47,49]$. Este tipo de activación es recomendable en caso de presentarse una rotación mesio lingual bilateral, ya que una activación en sentido mesio vestibular, además de rotar los molares, abre un espacio en mesial gracias a la forma romboidal de los molares dando la ilusión de un movimiento distal, lo que aumenta el perímetro del arco [47].

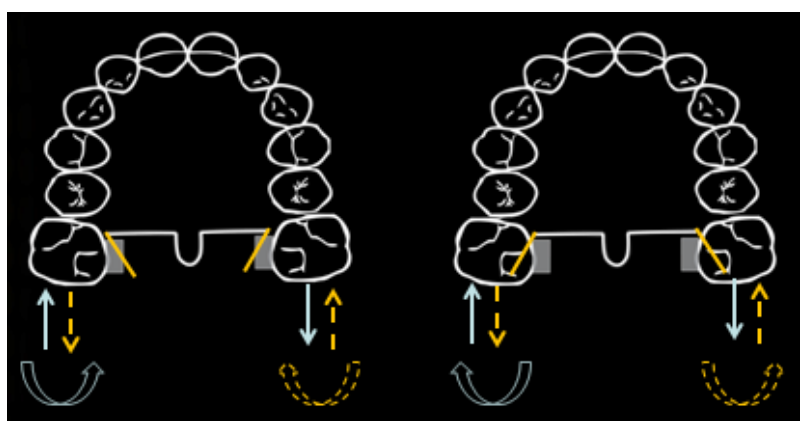

Figura 7. Activación simétrica en el plano transversal Fuente: elaboración propia

Detallada ya la activación simétrica, una activación asimétrica en el primer orden produce un momento asociado con la cupla, con fuerzas de equilibrio presentes en ambos molares en dirección anterior o posterior dependiendo de la activación del extremo de la BTP, las cuales siempre se registran en la cajuela que se encuentra ubicada en la superficie lingual de los molares; esto también genera momentos de poca magnitud asociados con las fuerzas, lo cual se considera insignificante clínicamente debido a que el punto de aplicación se encuentra muy cercano al centro de resistencia de los molares. Por ejemplo, al activar un extremo para una rotación mesio vestibular se produce un momento de cupla mesial vestibular en la cajuela, con fuerzas de equilibrio en dirección distal en el molar contralateral y en sentido mesial en el molar en el que se hizo la activación, con pequeños momentos asociados con las fuerzas de equilibrio en sentido mesio vestibular en el lugar de la activación y en sentido mesio lingual en el molar contralateral (figura 8). Si se realiza una activación en sentido mesio lingual, se dan los mismos momentos y las mismas fuerzas de equilibrio pero en sentido opuesto. En la medida que incremente el ángulo de entrada de un extremo de la barra en la cajuela, incrementa la magnitud del momento en el molar de la activación junto con las fuerzas de equilibrio y los momentos asociados en ambos molares.

Otro tipo de activación asimétrica en el primer orden puede ser un Step-bend, en el que se combinan dos activaciones unilaterales de las cuales se generan momentos en la misma dirección pero con fuerzas de equilibrio en sentido opuesto, y por tanto son una muy buena opción para incrementar la magnitud de las fuerzas de equilibrio sin la necesidad de aumentar el ángulo de entrada en las cajuelas. Como las fuerzas de equilibrio están en la misma dirección que el momento de la cupla, el momento de la fuerza en este caso se suma al momento de la cupla, así se considere despreciable, razón por la que este tipo de activación puede ser útil en la corrección de una relación molar clase II unilateral en la que se requieren fuerzas de gran magnitud en sentido distal [50].

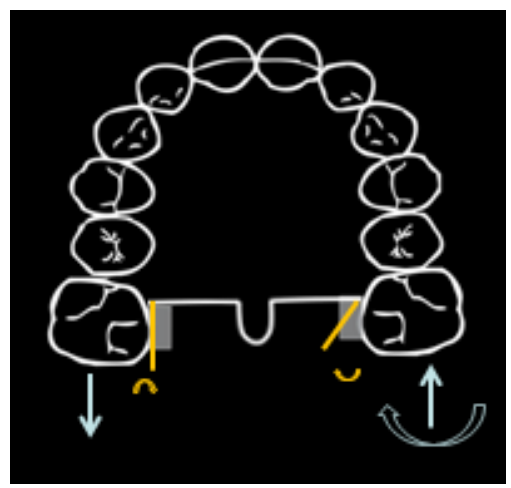

Figura 8. Activación asimétrica en el plano transversal Fuente: elaboración propia

\section{Activación en el segundo orden}

Las activaciones en el segundo orden resultan en una activación torsional del arco, ya que cuando los extremos son ubicados en las cajuelas un molar va a tener una tendencia a rotar en sentido horario mientras que el molar opuesto va a rotar en sentido antihorario. En este caso, los momentos en el plano sagital serán siempre iguales y opuestos porque la activación torsional del alambre hace que el ángulo de entrada sea siempre igual y opuesto entre los dos molares, y por tanto es imposible crear activaciones asimétricas, a no ser que uno de los molares se encuentre asociado con un sistema rígido. 


\section{Activaciones en el tercer orden}

Son similares a las activaciones del primer orden, sólo que en este caso se presentan en el plano frontal. Con la BTP se puede expandir o comprimir el arco superior cuando las fuerzas actúan en dirección bucal o lingual, respectivamente. Esto se debe a que las fuerzas son aplicadas en una situación coronal al centro de resistencia de los molares produciendo momentos rotacionales en dirección bucal o lingual que se pueden controlar [15, 47]. Por ejemplo, en una expansión se puede aplicar un torque radicular vestibular bilateral para que el movimiento del molar sea en cuerpo y no por inclinación, lo que favorece la estabilidad de la expansión y mejora el acople oclusal [51].

Una activación asimétrica en este plano puede resultar en un momento radicular vestibular o radicular lingual con fuerzas de equilibrio en sentido vertical (intrusivas-extrusivas), que se dan en el punto de aplicación de la fuerza. Por ejemplo, si a un solo molar se le aplica un torque radicular vestibular (equivale a un off center bend), sobre este actúa una fuerza de equilibrio intrusiva aplicada en la cajuela, mientras que en el molar contralateral se experimenta una fuerza de equilibrio extrusiva en la cajuela, en este caso las fuerzas de equilibrio pueden ser representativas porque se asocian con el momento de la cupla (figura 9).

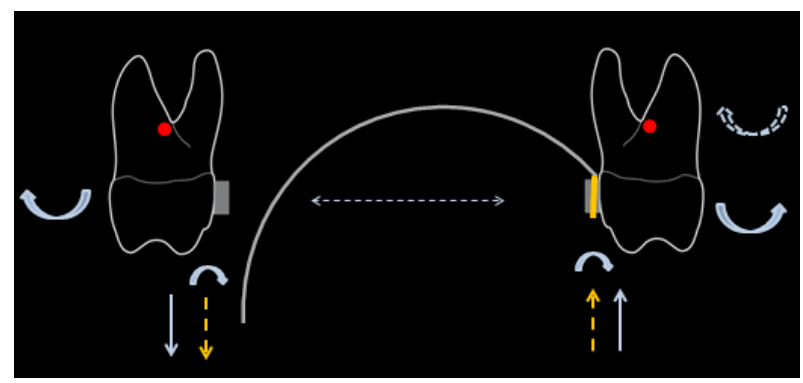

Figura 9. Activación asimétrica en el plano frontal

Fuente: elaboración propia

Una situación clínica en la cual la BTP es de gran ayuda, es en la corrección de una mordida cruzada posterior unilateral, en la que, al aplicar un torque radicular lingual en el molar que se encuentra en mordida cruzada en combinación con un torque radicular vestibular en el molar opuesto (equivale a un step bend), sumado a la fuerza expansiva de la BTP, puede causar un efecto más rápido en la inclinación bucal de la corona que un movimiento radicular del molar que sirve como anclaje. Con este tipo de mecánica, Ingervall et al. demostraron que es posible lograr una expansión significativa en el lado de la mordida cruzada sin mayor movimiento del molar que sirve como anclaje al darle torque radicular vestibular. La desventaja de este sistema son las fuerzas verticales de equilibrio resultantes con esta activación [43], en las que el molar con torque radicular vestibular tiende a intruirse, mientras que el que tiene un torque radicular lingual tiene un componente extrusivo (figura 10). Su aplicación es similar a la discutida en la activación unilateral de tercer orden, excepto por que con la adición del torque radicular lingual se puede corregir una mordida cruzada más rápidamente.

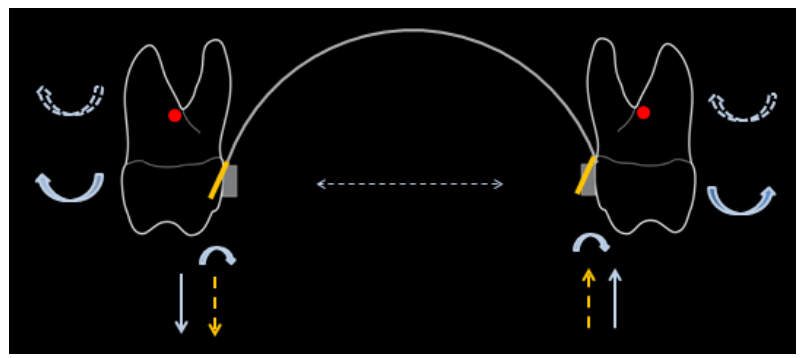

Figura 10. Step bend en el tercer orden

Fuente: elaboración propia

\section{Conclusiones}

Los sistemas parciales 4 x 2 y 6 × 2 ofrecen la oportunidad de trabajar con dos grupos de dientes que se encuentran separados por una gran distancia interbracket que, con base en una activación (off center bend), le permite al clínico la oportunidad de combinar dobleces de manera precisa en cualquier plano del espacio para ejercer el control molar favoreciendo la biomecánica durante el tratamiento de ortodoncia. Del mismo modo, la versatilidad de la barra traspalatina permite activaciones simétricas y asimétricas en distintos planos del espacio para controlar fuerzas y momentos para mantener o corregir la posición de los molares. Sin embargo, el hecho de no conocer o de no hacer una evaluación crítica del sistema, hace que cualquier activación pueda resultar en una sorpresa clínica indeseable, situación que se pueden controlar gracias a la posibilidad de hacer un análisis estático que permita predecir la dirección, magnitud relativa de los momentos y fuerzas presentes en cualquier sistema descrito antes de iniciar el movimiento del molar. 


\section{Referencias}

[1] Krishnan V, Ambili R, Davidovitch Z, Murphy N. Gingiva and orthodontic treatment. Semin Orthod. 2007; 13: $257-71$.

[2] Shroff B, Siegel SM. Treatment of patients with asymmetries using asymmetric mechanics. Semin Orthod. 1998; 4(3): 165-79.

[3] Dowsing P, Sandler PJ. How to effectively use a 2 x 4 appliance. J Orthod. 2004; 31(3): 248-58.

[4] Van Steenbergen E, Nanda R. Biomechanics of orthodontic correction of dental asymmetries. Am J Orthod Dentofacial Orthop. 1995; 107(6): 618-24.

[5] Smith RJ, Burstone CJ. Mechanics of tooth movement. Am J Orthod. 1984; 85(4): 294-307.

[6] Mulligan TF. Molar control. Part 1. J Clin Orthod. 2002; 36(1): 11-23.

[7] Andersen KL, Pedersen EH, Melsen B. Material parameters and stress profiles within the periodontal ligament. Am J Orthod Dentofacial Orthop. 1991; 99(5): 427-40.

[8] Tanne K, Sakuda M, Burstone CJ. Three-dimensional finite element analysis for stress in the periodontal tissue by orthodontic forces. Am J Orthod Dentofacial Orthop. 1987; 92(6): 499-505.

[9] Melsen B, Cattaneo P, Dalstra M, Kraft D. The importance of force levels in relation to tooth movement. Semin Orthod. 2007; 13: 220-33.

[10] Reitan K. Some factors determining the evaluation of forces in orthodontics. Am J Orthod. 1957; 43: 32-45.

[11] Krishnan V, Davidovitch Z. Cellular, molecular, and tissue-level reactions to orthodontic force. Am J Orthod Dentofacial Orthop. 2006; 129(4): 469 e1-32.

[12] Isaacson RJ, Lindauer SJ, Rubenstein LK. Activating a 2 x 4 appliance. Angle Orthod. 1993; 63(1): 17-24.

[13] Burstone CJ. Rationale of the segmented arch. Am J Orthod. 1962; 48: 805-22.

[14] Burstone CJ, Koenig HA. Creative wire bending. The force system from step and V bends. Am J Orthod Dentofacial Orthop. 1988; 93(1): 59-67.

[15] Isaacson RJ, Rebellato J. Two-couple orthodontic appliance systems: torquing arches. Semin Orthod. 1995; 1(1): 31-6.

[16] Proffit W. Contemporary orthodontics. 3a. ed. St Louis, Mo: Mosby; 2000.

[17] Rebellato J. Two-couple orthodontic appliance systems: activations in the transverse dimension. Semin Orthod. 1995; 1(1): 37-43.

[18] Burstone CJ, Koenig HA. Force systems from an ideal arch. Am J Orthod. 1974; 65(3): 270-89.
[19] Burstone CR. Deep overbite correction by intrusion. Am J Orthod. 1977; 72(1): 1-22.

[20] Mulligan TF. Understanding and applying wire-bracket angles. J Clin Orthod. 2008; 42(10): 563-73; quiz 95.

[21] Mulligan TF. Common Sene Mechanics in Everyday Orthodontics. CSM: Phoenix; 2009.

[22] Mulligan TF. Molar control. Part 5. J Clin Orthod. 2002; 36(5): 285-90.

[23] Hart A, Taft L, Greenberg SN. The effectiveness of differential moments in establishing and maintaining anchorage. Am J Orthod Dentofacial Orthop. 1992; 102(5): 434-42.

[24] Mulligan TF. Molar control. Part 4. J Clin Orthod. 2002; 36(4): 237-46.

[25] Lamons F, Holmes C. The problem of the rotated maxillary first permanent molar. Am J Orthod. 1961; 47(4): 246-72.

[26] Stoller A. The normal position of the maxillary first permanent molar. Am J Orthod. 1954; 40(4): 259-71.

[27] Andrews LF. The six keys to normal occlusion. Am J Orthod. 1972; 62(3): 296-309.

[28] Carlon JA. Rotation of the 1st maxillary molar. Inf Orthod Kieferorthop. 1973; 5(2): 137-62.

[29] Mulligan TF. Molar control. Part 2. J Clin Orthod. 2002; 36(2): 67-78.

[30] Mulligan TF. Molar control. Part 3. J Clin Orthod. 2002; 36(3): 147-58; quiz 6.

[31] Cobo JM, Diaz B, de Carlos F. Maintaining anchorage with a combination Nance-Goshgarian transpalatal arch. J Clin Orthod. 1998; 32(11): 681.

[32] Zachrisson BU. Clinical use of custom-made transpalatal arches. Why and how. World J Orthod. 2004; 5(3): 260-7.

[33] Ziegler P, Ingervall B. A clinical study of maxillary canine retraction with a retraction spring and with sliding mechanics. Am J Orthod Dentofacial Orthop. 1989; 95(2): 99-106.

[34] Stivaros N, Lowe C, Dandy N, Doherty B, Mandall NA. A randomized clinical trial to compare the Goshgarian and Nance palatal arch. Eur J Orthod. 2010; 32(2): 171-6.

[35] Dahlquist A, Gebauer U, Ingervall B. The effect of a transpalatal arch for the correction of first molar rotation. Eur J Orthod. 1996; 18(3): 257-67.

[36] Cetlin NM, Ten Hoeve A. Nonextraction treatment. J Clin Orthod. 1983; 17(6): 396-413.

[37] Ten Hoeve A. Palatal bar and lip bumper in nonextraction treatment. J Clin Orthod. 1985; 19(4): 272-91.

[38] Mandurino M, Balducci L. Asymmetric distalization with a TMA transpalatal arch. J Clin Orthod. 2001; 35(3): 174-8. 
[39] Kucher G, Weiland FJ. Goal-oriented positioning of upper second molars using the palatal intrusion technique. Am J Orthod Dentofacial Orthop. 1996; 110(5): 466-8.

[40] Wise JB, Magness WB, Powers JM. Maxillary molar vertical control with the use of transpalatal arches. Am J Orthod Dentofacial Orthop. 1994; 106(4): 403-8.

[41] Chiba Y, Motoyoshi M, Namura S. Tongue pressure on loop of transpalatal arch during deglutition. Am J Orthod Dentofacial Orthop. 2003; 123(1): 29-34.

[42] Deberardinis M, Stretesky T, Sinha P, Nanda RS. Evaluation of the vertical holding appliance in treatment of high-angle patients. Am J Orthod Dentofacial Orthop. 2000; 117(6): 700-5.

[43] Ingervall B, Gollner P, Gebauer U, Frohlich K. A clinical investigation of the correction of unilateral first molar crossbite with a transpalatal arch. Am J Orthod Dentofacial Orthop. 1995; 107(4): 418-25.

[44] Gollner P, Bantleon HP, Ingervall B. Force delivery from a transpalatal arch for the correction of unilateral first molar cross-bite. Eur J Orthod. 1993; 15(5): 411-20.

[45] Burstone CJ, Koenig HA. Precision adjustment of the transpalatal lingual arch: computer arch form predetermination. Am J Orthod. 1981; 79(2): 115-33.
[46] Yoshida N, Koga Y, Jost-Brinkmann PG, Kobayashi K. Clinical measurement of force systems upon activation of transpalatal arch in the treatment of unilateral crossbite. Prog Orthod. 2003; 4(2): 50-60.

[47] Rebellato J. Two-couple orthodontic appliance systems: transpalatal arches. Semin Orthod. 1995; 1(1): 44-54.

[48] Gunduz E, Zachrisson BU, Honigl KD, Crismani AG, Bantleon HP. An improved transpalatal bar design. Part I. Comparison of moments and forces delivered by two bar designs for symmetrical molar derotation. Angle Orthod. 2003; 73(3): 239-43.

[49] Ingervall B, Honigl KD, Bantleon HP. Moments and forces delivered by transpalatal arches for symmetrical first molar rotation. Eur J Orthod. 1996; 18(2): 131-9.

[50] Eyüboğlu S, Bengi A, Gürton A, Akin E. Asymmetric Maxillary First Molar Distalization with the Transpalatal Arch. Turk J Med Sci. 2004; 34(1): 59-66.

[51] Haas S, Cisneros G. The Goshgarian Transpalatal bar: A Clinical and an Experimental Investigation. Semin Orthod. 2000; 6: 98-105. 